\title{
Trigger Tool Based Detection of Adverse Drug Reactions in a Tertiary Care Teaching Hospital: A Prospective Observational Study
}

\author{
Raja Sree Gadde', Divya Teja Dhanenkula', Sowjanya Kammila', Dharani Nelluri', Manjusha \\ Polisetty ${ }^{1}$, Sowmya Kaniganti ${ }^{3}$, Yamini Vadlamannati ${ }^{3}$, Siva Reddy Challa ${ }^{2}$, Lakshmi Pavani Anne ${ }^{1}$, \\ Krishna Sri Nalla ${ }^{2}$, Sravya Gethika Dammalapati ${ }^{1}$
}

'Department of Pharmacy Practice, KVSR Siddhartha College of Pharmaceutical Sciences, Vijayawada-520 010, Andhra Pradesh, INDIA.

${ }^{2}$ Department of Pharmacology, KVSR Siddhartha College of Pharmaceutical Sciences, Vijayawada-520 010, Andhra Pradesh, INDIA. ${ }^{3}$ Department of Pharmacology, Dr. Pinnamaneni Siddhartha Institute of Medical Sciences and Research Foundation, Chinoutpalli, Gannavaram, Krishna -521286, Andhra Pradesh, INDIA.

\begin{abstract}
Background: The use of "triggers" to identify Adverse Drug reactions (ADRs) is a novel emerging method for measuring the overall level of harm from medications in a health care organization. Our main objective is to determine the incidence of adverse drug reactions in the hospitalized patients and to compare Global Trigger Tool (GTT) with conventional method to identify ADRs. Methodology: A Prospective observational study was conducted over a period of six months during November 2016-April 2017. Modified Global Trigger Tool was used to identify triggers. 16 triggers were used to identify ADRs. Causality assessment ofADRs was done using Naranjo scale and severity and harm categorization of ADRs were assessed using NCC MERP. Results: A total of 244 patient profiles were analyzed. The results reveal that 193 triggers were identified in 125 patients and 93 ADRs were found in 81 patients. Out of which, 64(68.81\%) ADRs were found by triggers and 29(31.18\%) ADRs were found spontaneously without the presence of a trigger. There is a remarkable improvement in the identification of ADRs using trigger tool in comparison to traditional approach. Of 93 ADRs identified, 69 (74.19\%) were probable and $24(25.81 \%)$ were possible. Similarly, 65 (69.89\%) were determined to be NCC-MERP harm category E and $28(30.11 \%)$ were category F. Conclusion: The study results suggest that IHI global trigger tool could be useful to identify ADRs in hospitals twice as more efficiently when compared to traditional ADR identification methods. It is an effective method to enable clinical pharmacists to identify ADRs and management of the same.
\end{abstract}

Key words: Adverse drug reactions (ADRs), IHI global trigger tool, NCC-MERP harm category, Causality, Prospective, Naranjo scale.

\section{INTRODUCTION}

Adverse drug reactions (ADRs) are one of the leading causes of morbidity and mortality. It has been estimated that approximately $2.9 \%-5.6 \%$ of all hospital admissions are caused by ADRs and as $35 \%$ of the hospitalized patient's experience an ADR during their hospital stay. ${ }^{1}$ ADR incidence has been reported in the range of 5.9 to $22.3 \%$ of all emergency department admissions in India. It has been reported that deaths due to ADRs contributed for $1.8 \%$ of total of deaths in India. ${ }^{2}$ Early detection, evaluation and monitoring of ADRs are essential to reduce harm to patients and thereby improving public health. ${ }^{3}$ The detection of ADRs has become increasingly significant because of the introduction of many newer medicines in the last two or three decades.

A trigger is defined as an occurrence, prompt or flag found on review of the medical record that "triggers "further investigation to determine the presence or absence of an adverse event". ${ }^{4}$ A trigger may include laboratory trig-
DOI: 10.5530/ijopp.11.2.18

Address for correspondence:

Dr. Siva Reddy Challa, M.Pharm, Ph.D, Professor and HOD, Department of Pharmacology, KVSR Siddhartha College of Pharmaceutical Sciences, Pinnamaneni Poly Clinic Road, Siddhartha NagarVijayawada-520010, Andhra Pradesh, INDIA. Phone no: +918008593009 Email Id: sivareddypharma@gmail.com

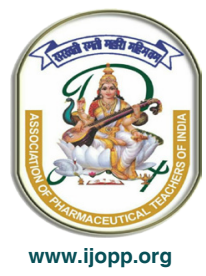


ger, medical trigger and clinician trigger. Earlier studies report that use of triggers promotes more focused chart review and thus may help to identify ADRs. ${ }^{4,5,6}$ The Institute of Healthcare Improvement (IHI) simplified the manual patient case chart review process and developed the Global Trigger Tool (GT'T) consisting of 19 triggers in order to monitor adverse events rates in a way that was easy to replicate in hospitals, with or without computerized records. ${ }^{7}$

Recent studies using the IHI Global Trigger Tool have identified harm rates in adults in US hospitals of 49 per 100 Admissions (33\% of patients), 86 per 100 admissions (28\% of patients) in Medicare patients, 25 per 100 admissions ( $18 \%$ of patients) across North Carolina. ${ }^{9}$ Till date, there are very few studies conducted in this area using trigger tool approach. Hence, our study aimed to study the assessment of ADRs using trigger tool approach and conventional method.

\section{METHODOLOGY}

\section{Study design and Participants}

This is a prospective observational study which was carried out for a period of 6 months November 2016 to April 2017) in patients admitted to Dr. Pinnamaneni SiddharthaInstitute of Medical Sciences and Research Foundation, which is an 850-bedded tertiary care teaching hospital at Chinaoutpalli, Gannavaram Mandal, Krishna district, Andhra Pradesh (India).

\section{Ethical consideration}

The study protocol (Number: PG/160/2017) was approved by Institutional Ethics Committee of Dr. Pinnamaneni Siddhartha Institute of Medical Sciences \& Research Foundation (Dr. PSIMS \& RF) which was registered with CDSCO (Reg. No: ECR/804/Inst/ AP/2016). All the participants were informed about study details and informed consent was obtained before the initiation of study.

\section{Inclusion criteria}

i) Patients with age greater than 18 years old ii) Patients agreed to participate voluntarily with written consent form iii) Patients who were admitted as inpatients in the study duration.

\section{Exclusion criteria}

i) Patients who were hospitalized less than 48 hours ii) Patients admitted to pediatrics and gynecology ward.

\section{Study Procedure}

A total of 244 patients, who met the inclusion criteria, were recruited into the study. A suitable data collection form was designed for use in the study. The sources of data were patient case sheets and laboratory data. All the recorded data was reviewed independently to identify 'triggers' and when a trigger was found, patient record was investigated in depth to determine whether an ADR occurred. If an ADR was discovered incidentally when going through the patient charts, without the presence of a specific trigger, this ADR was also considered and recorded as a "non-triggered" or "spontaneous" ADR, in accordance with the IHI methodology.

Harm categorization and causality were assessed for observed ADRs using National Coordinating Council for Medication Error Reporting and Prevention Index (NCC MERP) and Naranjo scale respectively. Positive Predictive Value (PPV) was calculated for each trigger as, number of ADRs identified with the trigger/number of triggers found in the patient charts.

\section{Identification of Triggers}

The (IHI) simplified the manual medical record review process and developed (GTT) consisting of 19 triggers to monitor adverse events rates in a way that was easy to replicate in hospitals, with or without computerized records. ${ }^{7}$ But, in our study, modified IHI trigger tool consisting of 16 triggers was used. List of modified IHI global triggers were presented in Table 1.

\section{Clinical Outcomes}

The primary outcome was to assess incidence of ADRs using trigger tool and traditional approach. The secondary outcome was to identify the factors associated with them.

\section{RESULTS}

During the study period, a total of 244 patients were admitted into the hospital. Of which, 193 triggers were identified in 125 patients and 93 ADRs were found in 81 patients. Out of which 64 ADRs (68.81\%) were found by triggers and 29 ADRs (31.18\%) were found spontaneously without the presence of a trigger. Out of 81 patients with ADR's, [45 (55.56\%)] were females and [36 (44.44\%)] were males (Figure 1).

The frequency distribution of triggers used to identify suspected ADRs were as follows. Laboratory triggers accounted for [34 (36.55\%)] followed by medication triggers contributed for [27 (29.03\%)] and clinical triggers [3 (3.22\%)] (Figure 1).

The Naranjo probability scale was applied to the study participants to determine the strength of the causal relationship with the drugs used by the patients and implicated in the occurrence of each ADR. The results of degree of causality assessment were as follows. Of 93 total ADRs, $69(74.19 \%)$ were probable while 24 
Table 1: List of modified IHI Global Triggers followed in the study.

\begin{tabular}{|c|c|}
\hline T1-Abrupt Medication Stop & T2-Glucose Less than $50 \mathrm{mg} / \mathrm{dl}$ \\
\hline T3-Anti-Emetic Administration & T4-Vitamin K Administration \\
\hline T5-International Normalized Ratio (INR) Greater than 6 & $\begin{array}{l}\text { T6-Rising BUN or Serum Creatinine Two Times (2X) over } \\
\text { Baseline }\end{array}$ \\
\hline T7-Rash & T8-Antidiarrheals \\
\hline T9-WBC count $<3000$ cells/cu.mm & T10-Elevated ALT/AST levels \\
\hline T11-Hypokalemia & T12-Hyperkalemia \\
\hline T13-Hyponatremia & T14-Decrease in Haemoglobin or Haematocrit of $25 \%$ or Greater \\
\hline T15-Platelet Count Less than 50,000 & T16-Hypotension \\
\hline
\end{tabular}

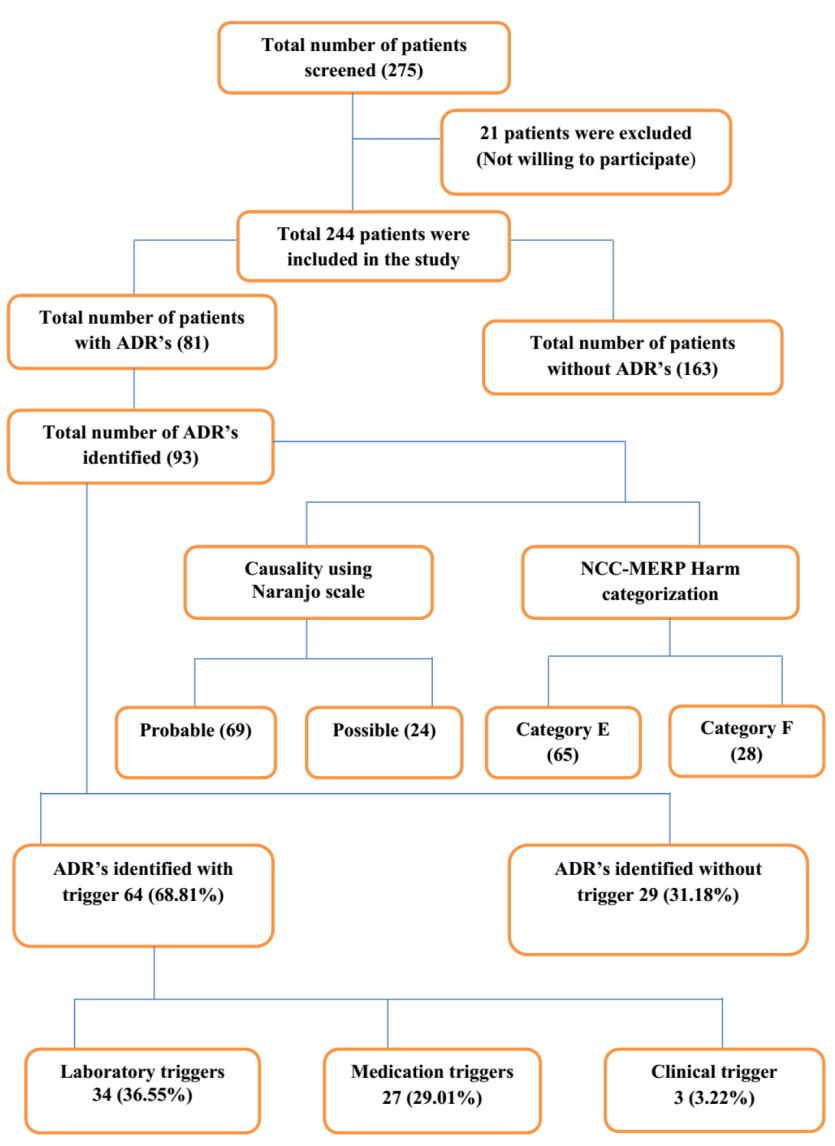

Figure 1: An illustration of study design and summarized results.

(25.81\%) were possible the severity of harm of every ADR was scored using categories $E$ to I of the National Coordinating Council for Medication Error Reporting and Prevention (NCC - MERP) severity scoring scale. The patients accounted for severity of ADRs were, 65 $(69.89 \%)$ of category E, $28(30.11 \%)$ of category F. Harm category $\mathrm{E}$ are more than harm category $\mathrm{F}$ (Figure 1).

Hypokalemia $(21.50 \%)$ trigger has most frequently observed followed by the anti-emetics (13.97\%), laxatives $(9.67 \%)$, decreased hemoglobin $(3.22 \%)$, hyponatremia $(3.22 \%)$, hyperkalemia $(3.22 \%)$, rash $(3.22 \%)$, serum glucose $<50 \mathrm{mg} / \mathrm{dl}(2.15 \%)$, vitamin $\mathrm{K}(2.15 \%)$, INR $>6$
(1.07\%), anti-diarrheal agents (1.07\%), elevated AST/ ALT levels (1.07\%), platelet count $<50,000(1.07 \%)$ Positive predictive value (PPV) was found to be higher (1) for the triggers "Serum glucose $<50 \mathrm{mg} / \mathrm{dl}$ ", "Rash" and "Platelet count $<50,000$ ". Next higher PPV of 0.67 was observed for the trigger "hypokalemia". This is followed by PPV of 0.57 for anti-emetics trigger and INR $>6$, cough suppressants. The data was shown in Table 2.

The descending order of organ systems affected by ADRs was as follows. Gastrointestinal system (GI, $43.01 \%)>$ cardiovascular $(29.03 \%)>$ Hematological (9.67\%), Endocrine (4.30\%), Respiratory (4.30\%), Dermatological (3.22\%) and Hepatic, Renal and Ophthalmic system (2 each, 2.15\%) (Table 3).

\section{DISCUSSION}

The incidence of ADRs observed in our study period $(38.1 \%)$ is remarkably higher in comparison to the incidence of other studies using different traditional methods. Our results are supported by similar previous research findings. A study conducted at a teaching hospital in Belgium by Carnevali et al. reported 25\% incidence of ADRs. ${ }^{10}$ Nonetheless, the incidence of ADRs in our study is lower than other study where the incidence was found to be $41 \%{ }^{11}$

Approximately $2 / 3^{\text {rd }}(68.81 \%)$ of ADRs were detected by triggers and $1 / 3^{\text {rd }}(31.18 \%)$ of ADRs were found spontaneously without the presence of a trigger. It implies that number of ADRs identified by trigger tool method is remarkably increased in comparison to the conventional method does. Our result is further supported by numerous studies reported that trigger tool method is more effective than conventional approach. ${ }^{12,13}$

Our study results also indicated that laboratory triggers were more contributed to detect ADRs followed by medication triggers and clinical triggers while a study conducted by Ganachari et al. (2013) indicated that suspected ADRs were identified majorly by medication 
Table 2: Positive predictive value (PPV) (effectiveness) of the triggers applied to identify adverse drug reactions (ADRs) in patient records of hospitalized patients.

\begin{tabular}{|c|c|c|c|}
\hline Triggers & $\begin{array}{c}\text { Number of positive } \\
\text { triggers }(A)\end{array}$ & Number of ADRs (B) & PPV (B/A) \\
\hline Abrupt medication stop & 03 & 0 & 0 \\
\hline Serum glucose $<50 \mathrm{mg} / \mathrm{dl}$ & 02 & 02 & 1 \\
\hline Anti emetics & 23 & 13 & 0.57 \\
\hline Vitamin $\mathrm{K}$ & 06 & 02 & 0.33 \\
\hline INR $>6$ & 02 & 1 & 0.50 \\
\hline Rise in Serum creatinine & 03 & 0 & 0 \\
\hline Rash & 03 & 03 & 1 \\
\hline Anti-diarrheal drugs & 03 & 01 & 0.33 \\
\hline Laxatives & 26 & 09 & 0.35 \\
\hline WBC Count $<3000$ cells/cu.mm & 01 & 0 & 0 \\
\hline Elevated ALT/AST levels & 05 & 01 & 0.20 \\
\hline Hypokalemia & 30 & 20 & 0.67 \\
\hline Hyperkalemia & 17 & 03 & 0.18 \\
\hline Hyponatremia & 14 & 03 & 0.21 \\
\hline Decreased Hemoglobin (greater than $25 \%$ ) & 40 & 03 & 0.07 \\
\hline Platelet count $<50,000$ & 01 & 01 & 1 \\
\hline Hypotension & 10 & 0 & 0 \\
\hline Cough Suppressants & 04 & 02 & 0.50 \\
\hline
\end{tabular}

\begin{tabular}{|c|c|}
\hline Organ system effected & Number of ADR's \\
\hline Gastrointestinal system & $40(43.01 \%)$ \\
\hline Cardiovascular system & $27(29.03 \%)$ \\
\hline Hematological system & $09(9.67 \%)$ \\
\hline Endocrine system & $04(4.30 \%)$ \\
\hline Respiratory system & $04(4.30 \%)$ \\
\hline Dermatological system & $03(3.22 \%)$ \\
\hline Hepatic system & $02(2.15 \%)$ \\
\hline Ophthalmological system & $02(2.15 \%)$ \\
\hline Renal system & $02(2.15 \%)$ \\
\hline
\end{tabular}

triggers followed by laboratory trigger tools and clinical triggers. ${ }^{14}$

Most frequent trigger was hypokalemia $(21.50 \%)$ in our study followed by anti-emetics, laxatives, rash, hyperkalemia, hyponatremia, decreased hemoglobin greater than $25 \%$, serum glucose $<50 \mathrm{mg} / \mathrm{dl}$, vitamin $\mathrm{K}$ $(2.15 \%)$, INR $>6(1.07 \%)$, and few triggers resulted in no ADRs such as rise in serum creatinine, WBC count $<3000$ cells/cu.mm and hypotension. High frequent hypokalemia could probably be explained by higher utilization pattens of diuretics in cardiovascular patients of study population. However, PPV of hypokalemia was 0.67 . It is the second most frequent trigger useful to identify ADRs. On the other hand, "hypoglycemia, rash and platelet count less than 50,000" were found to have highest PPV. Trigger tool approach was more efficient in identifying ADRs such as skin rash, blood sugar $<50 \mathrm{mg} / \mathrm{dl}$ and platelet count $<50,000$. Our results were also similar to the findings by Rozenfeld et al. (2013) wherein triggers like skin rash and blood sugar $<50 \mathrm{mg} / \mathrm{dl}$ were identified in $1 / 3^{\text {rd }}$ of adverse events. ${ }^{15}$ On the contrary, Naessens et al. resulted in the maximum probability of anti-emetic (32\%) trigger followed by the Diphenhydramine (10\%), abrupt medication stop ( $8 \%$ ), transfer to higher level of care $(4.9 \%)$, over-sedation/ Hypotension (3.8\%), Vitamin K administration (3.2\%), high serum creatinine $(2.6 \%)$ and glucose less than 50 $\mathrm{mg} / \mathrm{dl}(2.2 \%){ }^{16}$

Causality assessment results implied that probable ADRs accounts for $74.17 \%$ while possible contributed for $25.81 \%$. Our study showed similarity with the study by Arulmani et al. (2008) wherein $102(62.2 \%)$ reactions were assessed to be probable, $52(31.7 \%)$ as possible and $10(6.1 \%)$ as definite. ${ }^{17}$ NCC MERP scale was used for assessing the severity of ADRs. Category E ADRs accounted for $69.89 \%$ while Category F contributed for $30.11 \%$ which was in concordance with the study 
conducted by Naessens et al. (2010) wherein most of ADEs falls under category E. ${ }^{16}$ On the other hand, our study was in contrast to Rutberg et al. (2014) study reported that more ADEs under category $\mathrm{F}^{18}$ The organ system is most affected by ADRs in our study was gastrointestinal system followed by cardiovascular system. Our results were like the previous study conducted by Ganachari et al. wherein cardiovascular system is most affected followed by endocrine and neurological systems. ${ }^{14}$

\section{CONCLUSION}

Improved safety for patients is a universal priority in health care. However, efforts to impact meaningfully on safety and to reduce harm have been slowed by methodologies that fail to identify and quantify relevant clinical mishaps accurately. This study draws attention to the problem of ADRs in hospitalized patients and offers a methodological alternative by using modified IHI trigger tool. Our results suggest that trigger tool may be useful to identify ADR in hospitals twice as more efficiently when compared to traditional ADR identification methods.

\section{ACKNOWLEDGEMENT}

We thank Prof. Devala Rao Garikapati, Principal for providing his continuous support during this work. We also thank Director General (Dr.C.NageswaraRao), Hospital Superintendent\& HOD, Dept. of General Medicine (Dr.N.V. Krishna Rao) and Principal (Dr. P. Satyanarayana Murthy) of Dr.Pinnamaneni Siddhartha Institute of Medical Sciences and Research Foundation for granting permission to do Pharm.D Project work and providing the facilities for completion of this work.

\section{ABBREVIATIONS}

IHI: Institute for Health care Improvement; GTT: Global Trigger Tool; ADR: Adverse Drug Reaction; ADE: Adverse Drug Event; NCC MERP: National Coordinating Council for Medication Error Reporting and. Prevention; BMJ: British Medical Journal; PPV: Positive Predictive Value: CDSCO: Central Drugs Standard Control Organization.

\section{CONFLICT OF INTEREST}

Authors declare that there are no conflicts of interest to declare.

\section{SUMMARY}

Trigger tool approach is highly effective in the identification of ADRs in comparison to traditional approach. This method enables health care professionals including pharmacists for easy identification and reporting of ADRs. This could further increase the reporting of observed ADRs.

\section{REFERENCES}

1. Baniasadi S, Fahimi F, Shalviri G. Developing an Adverse Drug Reaction Reporting System at a Teaching Hospital. Basic and Clinical Pharmacology and Toxicology. 2008;102(4):408-11.

2. Amrita P, Singh SP. Status of spontaneous reporting of adverse drug reaction by physicians in Delhi. J Pharm Pract. 2011;4(2):29-36.

3. Beijer HJ, De Blaey CJ. Hospitalizations caused by adverse drug reactions: a meta-analysis of observational studies. Pharma World Sci. 2002;24(2):46-54.

4. Classen DC, Metzger J. Improving medication safety: The measurement conundrum and where to start. Int J Qual Health Care. 2003;15(1):41-7.

5. Matlow AG, Cronin CM, Flintoft V, Nijssen-Jordan C, Fleming M, Brady-Fryer B, et al. BMJ Qual Saf. 2011;20(5):416-23.

6. Brenner S, Detz A, Lopez A, Horton C, Sarkar U. Signal and noise: Applying a laboratory trigger tool to identify adverse drug events among primary care patients. BMJ Qual Saf. 2012;21:670-5.

7. Griffin FA, Resar RK. IHI Global Trigger Tool for Measuring Adverse Events (2nd Edition). IHI Innovation Series white paper, Cambridge MA: Institute for Healthcare Improvement. 2009.

8. Classen DC, Resar R, Griffin F, Federico F, Frankel T, Kimmel N, et al. 'Global Trigger Tool' shows that adverse events in hospitals may be ten times greater than previously measured. Health Affairs (Millwood). 2011;30(4):581-9.

9. Landrigan CP, Parry GJ, Bones CB, Hackbarth AD, Goldmann DA, Sharek PJ. Temporal trends in rates of patient harm resulting from medical care. $N$ Engl J Med. 2010;363(22):2124-34.

10. Carnevali L, Krug B, Amant F, Van Pee D, Gerard V, De Bethune X, et al. Performance of the Adverse Drug Event Trigger Tool and the Global Trigger Tool for Identifying Adverse Drug Events: Experience in a Belgian Hospital. Ann Pharmacother. 2013;47(11):1414-9.

11. Asavaroengchai S, Sriratanaban J, Hiransuthikul N, Supachutikul A. Identifying adverse events in hospitalized patients using Global Trigger Tool in Thailand. Asian Biomedicine. 2009;3(5):545-50.

12. Rozich JD, Haraden CR, Resar RK. Adverse drug event trigger tool: A practical methodology for measuring medication related harm. Qual Saf Health Care. 2003;12(3):194-200.

13. Sarkar U, Lopez A, Maselli JH, Gonzales R. Adverse drug events in U S. Adult ambulatory medical care. Health Serv Res. 2011;46(5):1517-33.

14. Ganachari MS, Wadhwa T, Walli S, Disha AK, Aggarwal A. Trigger tools for monitoring and reporting of adverse drug reactions: A Scientific tool for efficient reporting. Open access scientific reports. 2013;2(4):1-5.

15. Rozenfeld S, Giordani F, Coelho S. Adverse drug events in hospital: Pilot study with trigger tool. Rev Saude Publica. 2013;47(6):1102-11.

16. Naessens JM, O’Byrne TJ, Johnson MG, Vansuch MB, McGlone CM, Huddleston JM. Measuring hospital adverse events: Assessing inter-rater reliability and trigger performance of the Global Trigger Tool. Int J Qual Health Care. 2010;22(4):266- 74.

17. Arulmani R, Rajendran SD, Suresh B. Adverse drug reaction monitoring in a secondary care hospital in South India. Br J Clin Pharmacol. 2008;65(2):210- 6.

18. Rutberg H, Risberg MB, Sjodahl R, Nordqvist P, Valter, L, Nilsson L. Characterizations of adverse events detected in a university hospital: A 4-year study using the Global Trigger Tool method. BMJ. 2014;4(5):e004879. 